\title{
Parity and time-reversal symmetry breaking, singular solutions, and Fresnel surfaces
}

\author{
R M Kiehn, G. P Kiehn, and J B Roberds \\ Department of Physics Universityof Houston, \\ Houston, Texas 77004 (Received 18 December 1989) \\ Phys Rev A, 43 , (1991) p. 5665
}

\begin{abstract}
The method of exterior differential systems is applied to the problem of finding propagating singular solutions to Maxwell s equations Fresnel ray and wave surfaces are computed for systems that are not restricted to the topology imposed by the symmetries of the Lorentz transformations. The methods distinguish the measurable polarization phase differences between global behavior in optically active and Faraday media. In addition, an example is presented for which time-reversal and parity symmetries are broken: the inbound polarization states propagate with speeds different from the outbound polarization states.
\end{abstract}

\section{INTRODUCTION}

This article focuses attention on the mathematical problem of obtaining singular solutions to Maxwell's equations of electromagnetism for systems that do not necessarily have the symmetries of the Lorentz equivalence class similar to the vacuum. The methods employed are those suggested by Cartan [1] in his studies of exterior differential systems. Maxwell's equations are considered to be a system of global differential forms specifying certain topological properties about a differentiable variety. An intrinsic, physical distinction is made between the qualities of intensity ( $\mathbf{E}$ and $\mathbf{B}$ ) and the quantities of excitation $(\mathbf{D}$ and $\mathbf{H}$ ), and 
no assumption is made about the existence of a global metric on the variety. The singular solutions obtained are viewed from the perspective of Luneburg and Fock [2] as being those point set domains upon which discontinuities in field amplitudes may exist. Propagating discontinuities are interpreted as signals. A dynamical perspective and/or the presence of matter is presumed through the existence of a constitutive tensor density $\chi^{\mu \nu \alpha \beta}$ [3] which acts as a geometrical constraint on the topology induced by the Maxwell system of forms. The symmetries and antisymmetries of the constitutive map lead to an explicit extension of the concepts of the Fresnel wave normal surface and the Fresnel ray surface, historically developed for anisotropic birefringent media. [4] Explicit formulas for these surfaces are created that permit the analysis of combinations of effects, such as optical activity, and Faraday phenomena, for which time reversal and parity symmetries are not maintained.[5] The results are applicable to problems in which the fourfold degeneracy of the Maxwell-Lorentz system is broken, an example of which is given by systems employing four-mode ring laser devices.[6] In such systems, the inbound wave speeds are different from the outbound wave speeds.

\section{EXTERIOR DIFFERENTIAL SYSTEMS}

Consider the terminology and engineering format of Sommerfeld [7] for the Maxwell electromagnetic system:

$$
\begin{aligned}
& \operatorname{curl} \mathbf{E}+\partial \mathbf{B} / \partial t=0, \quad \operatorname{div} \mathbf{B}=0 \\
& \operatorname{curl} \mathbf{H}-\partial \mathbf{D} / \partial t=\mathbf{J}, \operatorname{div} \mathbf{D}=\rho
\end{aligned}
$$

Note that this system of partial differential equations is a specific realization of a closed ideal of differential forms [8], $\Sigma=\{F, H, J\}$ on a four-dimensional variety. That is, Maxwell's equations are equivalent to closure statements of differential forms:

$$
\begin{gathered}
d F=0 \\
d H=J, \quad d J=0
\end{gathered}
$$

A distinction in the sense of deRham [9] is to be made between the "pair" 2 -form $F$ of intensities and the "impair" N-2 form $H$ of excitations. For dimensionality greater than 4 , the exterior representation (2) is preferred over 
the classical representation (1), but in all dimensions (1) remains a nested subset of (2). In the language of forms, the representation of Maxwell's equations given by (2) are not only naturally covariant with respect to all diffeomorphisms (Lorentz, Galilean, or any other transformation with a differentiable inverse), but are also well behaved in a retrodictive (pull-back) sense with respect to continuous (but not necessarily homeomorphic), topology changing irreversible transformations. [10] The recognition of this fact raises questions as to the predilection of present day physics to emphasize the Lorentz transformations. As will be demonstrated below, the importance of the Lorentz transformations resides not with the question of natural covariance of the Maxwell equations (for which Van Dantzig has stated correctly that the covariance is trivial, [11] but instead relates to the concept that singular solution set to Maxwell's equations are only covariant with respect to the linear Lorentz transformation (and the nonlinear Mobius transformation). [12] For the work presented herein, the smaller source free system of forms $\Sigma_{0}=\{F, H\},\{J=0\}$ will be treated as a closed ideal. The Cartan technique will be used to search for two systems of dual vector fields that define point sets which are complimentary in a union and intersection sense. This problem is equivalent to finding those point sets upon which the field amplitudes admit discontinuities, but still satisfy Maxwell's equations. In other words, the solutions to Maxwell's equations are not unique upon the singular point set.

\section{RAYS}

The first problem of vector fields involves the search for a set of contravariant fields $\mathrm{S}$ which annihilate the system of forms of the closed ideal in a union sense. These point sets are given by the vectors

$$
S=\{\mathbf{s}, 1\}
$$

which are associated vectors to the forms of the differential ideal $\left\{\Sigma_{0}\right\}$ and satisfy the equations

$$
i(S)\left\{\Sigma_{0}\right\}=0,
$$

or

$$
i(S) F=0, \quad i(S) H=0 .
$$


As $d F=0$ and $d H=0$ for the source free ideal $\Sigma_{0}$, these associated vectors [13] are also extremal vectors for the closed ideal, and are therefore equivalent to the characteristic set.

In three-vector symbolism, the characteristic system satisfies the equations,

$$
\begin{aligned}
& \mathbf{s} \times \mathbf{B}+\mathbf{E}=0, \quad \mathbf{s} \cdot \mathbf{E}=0, \\
& \mathbf{s} \times \mathbf{D}-\mathbf{H}=0, \quad \mathbf{s} \cdot \mathbf{H}=0,
\end{aligned}
$$

from which it is apparent that $\mathbf{s}$ has the direction of the Poynting vector (energy flux),

$$
\mathbf{s}=\Gamma \mathbf{E} \times \mathbf{H} .
$$

The three-vector $\mathbf{s}$ will be defined as the ray field. Note that these ideas are metric free and do not depend upon a constitutive constraint.

\section{WAVES}

The second problem of vector fields involves the search for a set of covariant fields $k$ which annihilate the system of forms of the closed ideal in an intersection sense. These point set are given by the vectors

$$
k=\{\mathbf{k}, \omega\}
$$

such that the l-form $k=\mathbf{k} \cdot d \mathbf{r}-\omega d t$ will have null intersections with the closed ideal of forms $\left\{\Sigma_{0}\right\}$; i.e.,

$$
k^{\wedge}\left\{\Sigma_{0}\right\}=0
$$

or

$$
k^{\wedge} F=0, \quad k^{\wedge} H=0 .
$$

These equations are dual to the preceding equations for $\mathbf{s}$, and can be rewritten as set

$$
\begin{aligned}
& \mathbf{k} \times \mathbf{E}-\omega \mathbf{B}=0, \quad \mathbf{k} \cdot \mathbf{B}=0, \\
& \mathbf{k} \times \mathbf{H}+\omega \mathbf{D}=0, \quad \mathbf{k} \cdot \mathbf{D}=0,
\end{aligned}
$$


from which it is apparent that $\mathbf{k}$ has the direction of the momentum flux $\mathbf{D} \times \mathbf{B}$. It is to be noted that these six independent equations for the covariant wave field $\mathbf{k}$ and the dual six independent equations for the contravariant ray field $\mathbf{s}$ are the usual sets of equations which define the point sets upon which field discontinuities may exist.[12] The contravariant and the covariant sets may be reparameterized by arbitrary factors. Two common schemes for $\mathrm{k}$ are the de Broglie scheme

$$
p=(h / 2 \pi) k=(h / 2 \pi)(\mathbf{k} \cdot d \mathbf{r}-\omega d t)=\mathbf{p} \cdot d \mathbf{r}-E d t
$$

and the index of refraction scheme

$$
n=k / \omega=(\mathbf{k} \cdot d \mathbf{r}-\omega d t) / \omega=(\mathbf{n} \cdot d \mathbf{r}-d t) .
$$

It is to be noted that in a space time of four dimensions, the characteristic vectors $S$ are associated vectors to the dual l-forms $k$ :

$$
i(S) k=0 .
$$

This result yields a reciprocal relationship between the index of refraction vector $\mathbf{n}$ and the ray vector $\mathbf{s}$,

$$
\mathbf{n} \cdot \mathbf{s}=1 .
$$

Geometrically speaking, the ray vectors $S^{\mu}$ in four dimensions have orthogonal wave compliments $k_{\mu}$. Dimensionally, the three-vector $\mathbf{s}$ is a velocity (the energy flow or group velocity) and the three-vector $\mathbf{n}$ is a reciprocal velocity (the reciprocal phase velocity).

It should be noted that the four-vectors $S^{\mu}$ can be reparameterized (multiplied by any function of $\mathrm{x}, \mathrm{y}, \mathrm{z}, \mathrm{t})$ and, as generators of one parameter groups of transformations, leave the differential ideal of forms $\left\{\Sigma_{0}\right\}$ invariant with respect to the transformations. The differential ideal forms a set of absolute invariants with respect to the characteristic vectors.

\section{CHARACTERISTICS AND THEIR INVARIANTS}

If it is assumed that the 2 -form $F$ is exact, then the 1 -form $A$ of potentials

$$
A=\mathbf{A} \cdot d \mathbf{r}-\phi d t
$$

may be adjoined to the differential ideal, and the characteristic vectors of $\left\{\Sigma_{0}\right\}$ are extremal vectors of the larger set $\{F, H, A\}$. 
It follows that combinations of the intersections of the sets, created via the exterior product, are relative integral invariants with respect to the transformations generated by the characteristic vectors. The characteristic vectors leave invariant certain topological properties of the system that are characterized by the period integrals of

$$
\begin{gathered}
\text { topological flux }=A, \\
\text { topological charge }=H, \\
\text { topological spin }=A^{\wedge} H, \\
\text { topological torsion }=A^{\wedge} F .
\end{gathered}
$$

The question arises as to how many characteristic vectors exist. Consider the 2-form $F$ as an antisymmetric matrix in four dimensions. Its rank must be 0 , 2 , or 4 . If the rank is 4 , then no characteristic vectors (eigenvectors with null eigenvectors) exist. Note that if $F$ is of rank 4 , then $F^{\wedge} F \neq 0$, from which it follows that $\mathbf{E} \cdot \mathbf{B} \neq 0$. The criterion of rank zero implies the trivial solution only. Hence, if characteristics of $F$ exist, $F$ is of rank 2, and there are two and only two characteristic vectors in four dimensions. It follows that the second Poincare invariant $\mathbf{E} \cdot \mathbf{B}=0$ on the characteristic set. Similar arguments may be made for the ( $\mathrm{N}-2)$-form $H$.

\section{CONSTITUTIVE RELATIONS - A GEOMETRICAL CONSTRAINT}

The system of equations satisfied by the characteristic vectors may be viewed as six independent equations in 12 unknowns, $\mathbf{D}, \mathbf{E}, \mathbf{B}, \mathbf{H}$, for a given ray vector s. As such, the system is insoluble. However, consider a geometrical constraint between the (N-2)-form $H$ and the 2-form $F$, given by the constitutive tensor density $\chi^{\mu \nu \alpha \beta}$. Assume that

$$
H^{\mu \nu}=\chi^{\mu \nu \alpha \beta} F_{\alpha \beta}
$$

Due to the antisymmetries of the forms, the 256 components of $\chi^{\mu \nu \alpha \beta}$ in four dimensions may be reduced to 36 distinct values, [14] a reduction which leads to the 6 vector formalism of Sommerfeld; namely, in matrix format 


$$
\begin{aligned}
& \mathbf{D}=[\epsilon] \circ \mathbf{E}+[\gamma] \circ \mathbf{B} \\
& \mathbf{H}=\left[\gamma^{\dagger}\right] \circ \mathbf{E}+\left[\mu^{-1}\right] \circ \mathbf{B} .
\end{aligned}
$$

The $6 \mathrm{X} 6$ constitutive matrix is assumed to be Hermitian for the nondissipative case considered herein.

The six constitutive equations given above may be used to eliminate half of the unknowns in the six equations for the characteristic system. For example, the elimination of $\mathbf{E}$ and $\mathbf{H}$ in the characteristic equations leaves a homogeneous system of six equations in six variables. The Cramers determinantal condition must be satisfied if a solution exists.

\section{FRESNEL RAY SURFACES}

To simplify the algebra, define a $3 \times 3$ completely antisymmetric matrix $\left[\mathbf{s}^{\times}\right]$in terms of the three components of the ray vector $\left\{s^{x}, s^{y}, s^{z}\right\}$ :

$$
\left[\mathbf{s}^{\times}\right]=\begin{array}{ccc}
0 & s^{z} & -s^{y} \\
-s^{z} & 0 & s^{x} \\
s^{y} & -s^{x} & 0
\end{array}
$$

Then the determinantal function $\mathcal{L}\left(s^{x}, s^{y}, s^{z}\right)$ on the characteristic system may be expressed in matrix format as

$$
\mathcal{L}\left(s^{x}, s^{y}, s^{z}\right)=\operatorname{det}\left(\left[\mu^{-1}\right]+\left[\mathbf{s}^{\times}\right][\gamma]-\left[\gamma^{\dagger}\right]\left[\mathbf{s}^{\times}\right]+\left[\mathbf{s}^{\times}\right][\epsilon]\left[\mathbf{s}^{\times}\right]\right)
$$

The Cramers compatibility argument is the statement that $\mathcal{L}=0$, which in geometrical terms defines an implicit hypersurface on the three-dimensional space of variables $\left(s^{x}, s^{y}, s^{z}\right)$ for fixed values of $\mathrm{x}, \mathrm{y}, \mathrm{z}$, and t. This hypersurface, $\mathcal{L}=0$, is the extension of the Fresnel ray surface to include not only birefringence in anisotropic media, but also optical activity, Faraday rotation, and Fresnel-Fizeau phenomena in combination. Although the coefficients of the constitutive matrix are complex, the constraint of hermiticity guarantees the reality of the function $\mathcal{L}$. The Fresnel ray surface is of fourth degree, but is necessarily symmetric about the origin of the variables $s^{x}, s^{y}$, and $s^{z}$, only for birefringent media.

It is to be remembered that the characteristic vectors $S^{\mu}$ are four-dimensional vectors and come in pairs. These vectors are compatible in a Lie bracket sense, and as they can be reparametrized arbitrarily, the pair may be used to create a two-dimensional subspace of space-time. 


\section{FRESNEL WAVE SURFACES}

Arguments similar to those applied to the solubility of the ray system may be applied to the wave system. That is, for given values of $n_{x}, n_{y}$, and $n_{z}$, the system of wave constraints may viewed as six equations in 12 unknowns. Use the six constitutive equations to eliminate $\mathbf{D}$ and $\mathbf{B}$ and thereby obtain a homogeneous system of six equations in 6 unknowns. The Cramers rule yields a determinantal function, $\mathcal{H}\left(n_{x}, n_{y}, n_{z}\right)$, that must vanish if solubility is to exist:

$$
\mathcal{H}\left(n_{x}, n_{y}, n_{z}\right)=\operatorname{det}\left([\epsilon]+[\gamma]\left[\mathbf{n}^{\times}\right]-\left[\mathbf{n}^{\times}\right]\left[\gamma^{\dagger}\right]+\left[\mathbf{n}^{\times}\right]\left[\mu^{-1}\right]\left[\mathbf{n}^{\times}\right]\right)
$$

In the three-dimensional space of variables $\left(n_{x}, n_{y}, n_{z}\right)$ the implicit hypersurface $\mathcal{H}=0$ is of fourth degree and creates an extension of the usual Fresnel wave surface to include not only anisotropic birefringence, but also electric and magnetic Faraday rotation, optical activity, and Fresnel-Fizeau phenomena in combination. In the case of birefringence, the quartic function $\mathcal{H}=0$ splits into two quadratic factors which are the usual representations of the Fresnel ellipsoids. The equivalent geometrical Fresnel analysis for optically active, Faraday media, or media exhibiting magneto-electric effects does not appear in the literature to our knowledge. The general Fresnel wave surfaces are distorted ellipsoids which do not have a center of symmetry. Hence an arbitrary line through the origin in reciprocal phase velocity space will intercept the Fresnel wave surface(s) in four distinct points representing four distinct phase velocities: two magnitudes in the outbound direction for each state of polarization, and two different velocities in the inbound direction, one for each state of polarization. As an example, an $n_{z}=0$ slice has been displayed in Fig. 1 for a Fresnel wave surface that combines both optical activity and Faraday rotation. The entire wave surface is displayed in Fig. 6.

The little appreciated result is that the light signal does not propagate with the same phase velocity inbound as outbound in systems that do not have the symmetries of the Lorentz system. These effects were experimentally verified in dual polarized ring laser experiments conducted by Sanders. [6]

Another example showing the different behavior of singular solutions is given by the simple experiment of measuring the polarization rotation of a beam of light as it passes through two tubes of either optically active material (a water solution of sugar) or Faraday material (He-Ne gas with an axial magnetic field). For similarly adjusted parameters, the degree of polarization rotation is the same for the first pass through each medium, each electric vector will be rotated by 
$\Phi$ degrees. [See Fig. 2(a).] However, reflect the light beams with a mirror and let them make a round trip through the optically active sample and through the Faraday sample. The polarization will return to its initial state in the optically active sample, but the Faraday sample will accumulate another increment of phase rotation such that the round-trip rotation is $2 \Phi$ for the Faraday media and 0 for the optically active solution [see Fig. 2(b)]. The discrete symmetry of time inversion ( $\mathrm{t}$ goes to - $\mathrm{t}$ on reflection) yields different results. This problem was first solved theoretically in the monograph by Post.[3]

\section{EXAMPLES}

Plots of numerical solutions for the Fresnel wave surfaces $\mathcal{H}=0$ are given in Figs. 3-6. The example in Fig. 3 displays the completely isotropic case, and represents the Lorentz equivalence class for which the speed of discontinuity propagation is the same in all directions for all states of polarization. The isotropic sphere has the quartic equation

$$
X=1-2\left(x^{2}+y^{2}+z^{2}\right)+\left(x^{2}+y^{2}+z^{2}\right)^{2}=0 .
$$

The isotropic "spherical" surface $r^{2}=1$ is not the usual sphere. Indeed, the isotropic sphere is a strange surface for every point of the "surface" is a critical point. Not only does the density function $\mathcal{H}$ vanish on the set of isotropic points, but also the gradient of $\mathcal{H}$ vanishes on this set. Classical surfaces obey the GaussWeingarten as-sumptions, which imply that the surface gradient is nowhere zero.

In the neighborhood of the surface outside the isotropic set $r^{2}=1$, the gradient of $\mathcal{H}$ points away from the origin. In the neighborhood of the surface inside the isotropic set, the gradient points in towards the origin. For the standard spherical surface, the gradient points in the same direction everywhere and does not go to zero on the surface. The isotropic set consists of a double spherical surface with distinct orientations. The effect of the constitutive tensor will be to split the isotropic degeneracy. The splitting can take place in two distinct ways, each of which, permits a complex number representation and retains a center of symmetry for the deformed double spheres. However, when both distinct splitting mechanisms are present, the degeneracy can be removed in such a way that there is no center of symmetry, and an irreducible quaternionic representation is necessary. These ideas are consistent [15] with group-theoretic expectations based on Klein's "Vierer" group $\{E, P, T, P T\}$. The example of Fig. 4 modifies the isotropic case to include optical activity. The Fresnel wave surface exhibits 
a center of symmetry, but the degeneracy of the double sphere in the isotropic case is removed. Analytically, the degenerate real surface is split into a complex number pair.

The example of Fig. 5 modifies the isotropic case to include Faraday phenomena. The Fresnel wave surface again exhibits a center of symmetry, but the degeneracy of the double sphere in the isotropic case is removed. Again a complex representation is indicated, but of a type different from that of Fig. 4. (See Ref. 16 for the differences between complex numbers and quaternions.)

The example of Fig. 6 modifies the isotropic case with a combination of Faraday rotation and optical activity. The Fresnel wave surface no longer exhibits a center of symmetry, and a quaternionic (or Dirac spin) representation is necessary. It should be noted that the length of the vector from the origin to the Fresnel wave surface is a measure of the reciprocal wave velocity. The "outbound" wave speeds are related to the "inbound" wave speeds by extending the vector from the origin in the op-posite direction. The intercept with each Fresnel surface is interpreted as a distinct state of polarization. For the example of Fig. 6, all four wave speeds are distinct. The inbound speed of light is different from the outbound speed of light.

\section{ELECTROMAGNETIC SHOCK WAVES}

Another viewpoint of the systems of singular equations would be to consider the system to be an overdetermined system of equations for the ray vector $\mathrm{s}$ in terms of the given values of $\mathbf{D}, \mathbf{E}, \mathbf{B}, \mathbf{H}$. From this point of view certain compatibility relations [17] must be satisfied for a unique solution to exist. The compatibility relations are equivalent to the constraint that on the singular set

$$
\mathbf{D} \circ \mathbf{E}-\mathbf{B} \circ \mathbf{H}=0 .
$$

That is, the first Poincare invariant must vanish. In the language of differential forms, then the differential ideal is constrained such that $F^{\wedge} F=0$ and $F^{\wedge} H=0$.

The constraints for the singular wave field may be manipulated to yield

$$
\mathbf{k} \circ \mathbf{k}-\omega^{2}(\mathbf{D} \circ \mathbf{B}) /(\mathbf{E} \circ \mathbf{H})=(\mathbf{k} \circ \mathbf{E})(\mathbf{k} \circ \mathbf{H}) /(\mathbf{E} \circ \mathbf{H})
$$

This equation must necessarily be satisfied if a solution is to exist. Now if it is further assumed that the assumed solution is to exist uniquely in the sense of Frobenius, the wave vector must be proportional to a gradient field $\mathbf{k}=\lambda \nabla \phi$. Then the necessary condition becomes 


$$
(\nabla \phi)^{2}-(\mathbf{D} \circ \mathbf{B}) /(\mathbf{E} \circ \mathbf{H})(\partial \phi / \partial t)^{2}=(\mathbf{k} \circ \mathbf{E})(\mathbf{k} \circ \mathbf{H}) /(\mathbf{E} \circ \mathbf{H})
$$

With the further constraint of either magnetic $(\mathbf{k} \circ \mathbf{H}=0)$ or electric $(\mathbf{k} \circ \mathbf{E}=0)$ isotropy, the necessary condition becomes the eikonal equation

$$
(\nabla \phi)^{2}-(\mathbf{D} \circ \mathbf{B}) /(\mathbf{E} \circ \mathbf{H})(\partial \phi / \partial t)^{2}=0 .
$$

According to Kateyev, such a result defines the propagation of "electromagnetic shock waves" [18] that can be interpreted as signals. The result also implies that the wave surface function satisfies a first-order, quadratic, partial differential equation with invariant coefficients and signature $(+++-)$. It has been shown by Fock [12] that the Lorentz transformation and the Mobius transforrnation are the only transformations that leave such a quadratic form invariant. The Lorentz transformation is the only linear transformation that preserves the signature of the singular solution set to Maxwell's equations, independent of the fact that the frame of reference be "inertial" or not. As pointed out by Hermann, Van Dantzig, and indirectly by many others, the significance of the Lorentz transformations does not reside with natural covariance of the Maxwell field equations, but instead is to be associated with the natural covariance of the singular solution sets to Maxwell's equations.

\section{ALGEBRAIC DETAILS}

A large class of interesting problems may be studied in terms of the reduced $6 \mathrm{X}$ 6 constitutive matrix:

$$
\chi=\left[\begin{array}{cc}
{[-\epsilon]} & {[\gamma]} \\
{\left[\gamma^{*}\right]} & {\left[\mu^{-1}\right]}
\end{array}\right]=\left[\begin{array}{cccccc}
-\epsilon_{11} & i \epsilon_{12} & -i \epsilon_{13} & i \gamma 1 & 0 & 0 \\
-i \epsilon_{12} & -\epsilon_{22} & i \epsilon_{23} & 0 & i \gamma 2 & 0 \\
i \epsilon_{13} & -i \epsilon_{23} & -\epsilon_{33} & 0 & 0 & i \gamma 3 \\
-i \gamma 1 & 0 & 0 & \mu_{11}^{-1} & 0 & 0 \\
0 & -i \gamma 2 & 0 & 0 & \mu_{22}^{-1} & 0 \\
0 & 0 & -i \gamma 3 & 0 & 0 & \mu_{33}^{-1}
\end{array}\right]
$$

The Fresnel wave surface condition, $\mathcal{H}=0$, is equivalent to the vanishing of the determinant of a Hermitian $3 \times 3$ matric in the reciprocal velocity space $\left(n_{x}, n_{y}, n_{z}\right)$. The matrix $[\mathcal{H}]$ has the format 


$$
[\mathcal{H}]=\left[\begin{array}{ccc}
A & 0 & 0 \\
0 & B & 0 \\
0 & 0 & C
\end{array}\right]+\left[\begin{array}{lll}
0 & c & b \\
c & 0 & a \\
b & a & 0
\end{array}\right]+\left[\begin{array}{ccc}
0 & i \chi & -i \beta \\
-i \chi & 0 & i \alpha \\
i \beta & -i \alpha & 0
\end{array}\right],
$$

with a determinant equal to

$$
\mathcal{H}=(A B C)-A\left(a^{2}+\alpha^{2}\right)-B\left(b^{2}+\beta^{2}\right)-C\left(c^{2}+\chi^{2}\right)+2(c b a-c \beta \alpha-\chi b \alpha-\chi \beta a)
$$

where

$$
\begin{gathered}
A=-\left(n_{y}^{2} \mu_{33}^{-1}+n_{z}^{2} \mu_{22}^{-1}\right)+\epsilon_{11} \\
B=-\left(n_{z}^{2} \mu_{11}^{-1}+n_{x}^{2} \mu_{33}^{-1}\right)+\epsilon_{22} \\
C=-\left(n_{x}^{2} \mu_{22}^{-1}+n_{y}^{2} \mu_{11}^{-1}\right)+\epsilon_{33} \\
a=n_{y} n_{z} \mu_{11}^{-1} \\
b=n_{z} n_{x} \mu_{22}^{-1} \\
c=n_{x} n_{y} \mu_{33}^{-1} \\
\alpha=n_{x}(\gamma 2+\gamma 3)+\epsilon_{23} \\
\alpha=n_{y}(\gamma 3+\gamma 1)+\epsilon_{31} \\
\alpha=n_{z}(\gamma 1+\gamma 2)+\epsilon_{12}
\end{gathered}
$$

For momentum flow along the $\mathrm{x}$ direction $\left(\mathbf{n}=n_{x}, 0,0\right)$, and for an isotropic media $\left(\epsilon_{11}=\epsilon_{22}=\epsilon_{33}=\epsilon, \quad \mu_{11}=\mu_{22}=\mu_{33}=\mu\right)$ to which is added a mixture of optical activity $(\gamma 1=\gamma 2=\gamma 3=\gamma)$ and Faraday effect $\left(\epsilon_{23} \neq 0, \epsilon_{13}=\epsilon_{12}=0\right)$, the Fresnel wave surface condition reduces to the equation

$$
\mathcal{H}\left(n_{x}, 0,0\right)=\left(\epsilon-\mu^{-1} n_{x}^{2}\right)^{2}-\left(\epsilon_{23}-2 \gamma n_{x}\right)^{2}=0 .
$$

The solutions imply four distinct values of $n_{x}$, and therefore four distinct phase velocities of propagation depending on the polarization and the propagation direction. This result was first obtained by Schultz et aL. [5] using a plane-wave vector potential analysis of Maxwell's equations. The work of Schultz et al. also gave vector wave (quaternionic) solutions as well as the phase velocities for the 
above example, but required a somewhat unclear rank reduction argument to obtain the desired result. From the work presented herein it is seen that the result is due to an application of Cramers's theorem on the existence of solutions applied to the singular solution set. Historically, the notion that the singular solution sets and wave fronts are related concepts is due to Fock and Luneburg, following suggestions made by Hadamard.[19]

\section{ACKNOWLEDGMENT}

This work was supported in part by the Energy Laboratory of the University of Houston.

*Present address: Imperial College, London, England.

\section{References}

[1] E. Cartan, Systems Differentials Exterieurs et leurs Applications Geometriques (Hermann, Paris, 1945).

[2] R. K. Luneburg, The Mathematical Theory of Optics (University of California Press, Berkeley, 1964).

[3] E. J. Post, The Formal Structure of Electromagnetics (North-Holland, Amsterdam, 1962).

[4] M. Kline and I. W. Kay, Electromagnetic Theory and Geometrical Optics (Interscience, New York, 1965).

[5] A. K. Schultz et al., Phys. Lett. 74A, 384 (1979).

[6] V. Sanders and R. M. Kiehn, IEEE J. Quantum Electron. QE-13, 739 (1977)

[7] A. Sommerfeld, Electrodynamics (Academic, New York, 1952).

[8] R. M. Kiehn and J. F. Pierce, Phys. Fluids 12, 1941 (1969).

[9] G. DeRham, Varietes Diffierentiables (Hermann, Paris, 1955).

[10] R. M. Kiehn, Int. Eng. Sci. 14, 749 (1976).

[11] D. Van Dantzig, Proc. Cambridge Philos. Soc. 30, 421 (1934).

[12] V. Fock, Space Time and Gravitation (McMilian, New York, 1964).

[13] J. Klein, Ann Inst. AIFUB Fourier, Grenoble 12, 1 (1962).

[14] See E. J. Post, The Formal Structure of Electromagnetics (Ref. 3), p. 132.

[15] It has been pointed out that these observations were made by $\mathrm{H}$. Walker in an unpublished personal communication. Also see Ref. 5. 
[16] L. Brand, Vector and Tensor Analysis (Wiley, New York, 1962).

[17] C Lanczos, Linear Differential Operators (Van Nostrand, London, 1961).

[18] C. Katayev, Electromagnetic Shock Waves (ILIFFE, Lon-don, 1966).

[19] J. Hadamard, Lectures on Cauchy's Problem in Linear Partial Differential Equations (Dover, New York, 1952), p. 52. 


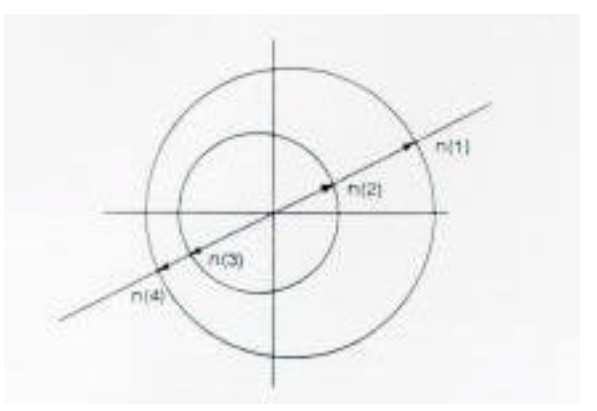

\section{Caption FIG. 1}

Fresnel ellipsoids determine the wave speeds for different directions and different states of polarizations: $\mathrm{n}(1)$ represents the index of refraction for polarization state 1 , outbound at $30^{\circ}$ in the xy plane; $\mathrm{n}(2)$ represents the index of refraction for the opposite polarization state, outbound; $\mathrm{n}(3)$ represents the first polarization state, inbound at $210^{\circ} ; \mathrm{n}(4)$ represents the second polarization state, inbound.

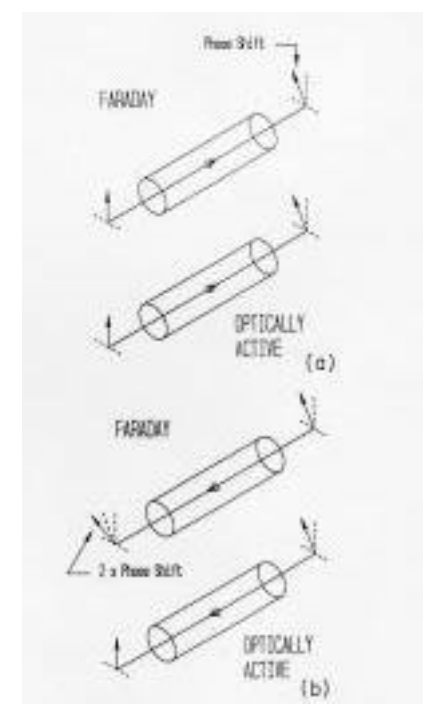

\section{Caption Fig. 2}

(a) Optical activity and Faraday rotation are not reciprocally equivalent. For suitably adjusted parameters, the rotation of the polarization vector is the same for one way traversal through Faraday or optically active media. (b) Upon reflection, the polarization phase shift returns to the original value for an optically active media, where the Faraday rotation accumulates an additional increment of phase shift. 


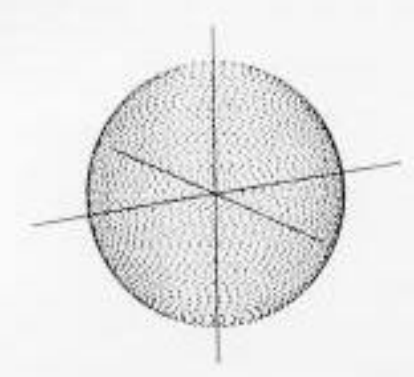

\section{Caption Fig. 3}

The Isotropic Fresnel wave surface $\mathcal{H}=0$ [see eq's (23) and (31)] consists entirely of critical points. All propagation speeds for all states of polarization are the same. This surface is not equivalent to a simple sphere, for the surface gradient vanishes everywhere . [Wave solution surface to Eq. (31) for $\epsilon=1, \mu=1, \gamma=0$.]
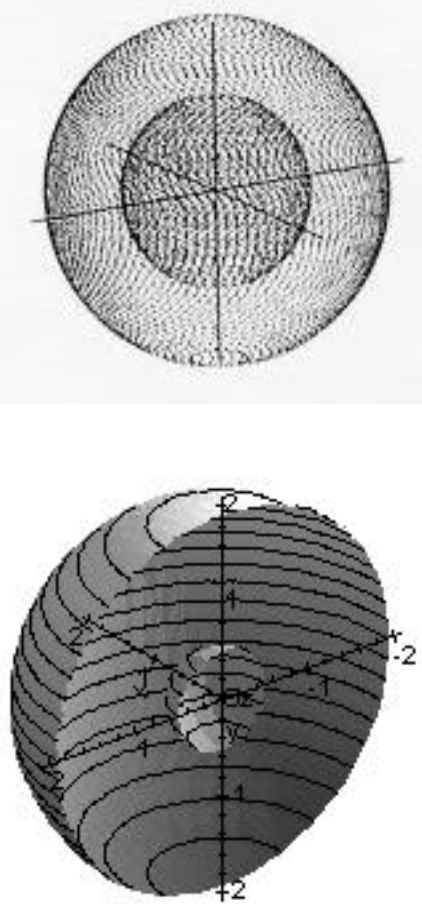


\section{Caption Fig 4.}

The Isotropic case is modified to include Optical Activity. A center of symmetry and isotropy is preserved, but chirality is broken. Inbound waves of the same chirality propagate with the same speed as outbound waves of the same chirality. [Wave solution surface to Eq. (31) for $\epsilon=1, \mu=1, \gamma=0.3$.] 


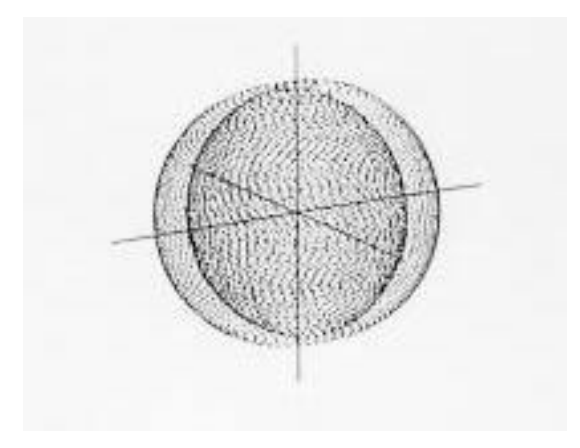

\section{Caption Fig. 5}

The Isotropic case is modified to include Faraday rotation. A center of symmetry is preserved, although isotropy is broken. This Fresnel wave surface is not equivalent to the Fresnel wave surface of Figure 4. [Wave solution surface to Eq. (31) for $\epsilon=1, \mu=1, \gamma=0, \epsilon_{12}=0.3$.]

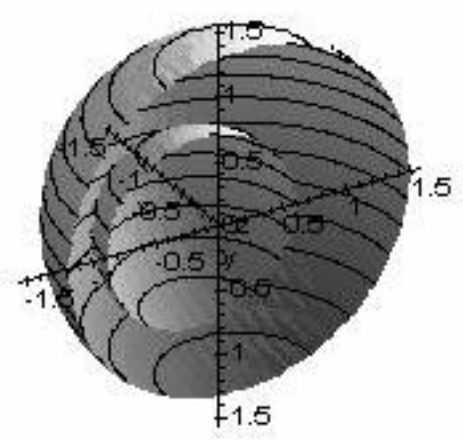

\section{Caption Fig 6.}

The Isotropic case is modified to include both Faraday rotation and Optical Activity. No longer does the Fresnel wave surface exhibit a center of symmetry. Inbound and outbound waves of different polarizations travel with (four) different speeds. [Wave solution surface to Eq. (31) for $\epsilon=1, \mu=1, \gamma=0.3, \epsilon_{12}=0.3$.] 